\title{
Test yourself: question and answer question: painful knee swelling
}

\section{Akarshan Monga $^{1} \cdot$ Andrew Rosenberg $^{2} \cdot$ Frank $^{\prime}$ Dea $^{3} \cdot$ John Durham $^{4} \cdot$ Ty K. Subhawong ${ }^{5}$ (D)}

Published online: 5 July 2019

(C) ISS 2019

\section{Question: Painful Knee Swelling.}

19 year old female with right knee pain for 7 years and increased knee swelling for 6 months (Figs. 1, 2, 3, 4, and 5).

The diagnosis can be found at https://doi.org/10.1007/s00256-01903274-1

Ty K. Subhawong

tsubhawong@miami.edu

1 Nova Southeastern University- College of Osteopathic Medicine, Davie, FL, USA

2 Department of Pathology, University of Miami Miller School of Medicine, 1400 NW 12th Avenue, Miami, FL 33136, USA

3 Health Sciences Centre, Memorial University of Newfoundland, 300 Prince Philip Drive, St. John's, NL A1B 3V6, Canada

4 Northern Arizona Orthopaedics, 1485 N. Turquoise Drive, Flagstaff, AZ 86001, USA

5 Department of Radiology, University of Miami Miller School of Medicine/Jackson Memorial Hospital, 1611 NW 12th Ave., JMH WW 279, Miami, FL 33136, USA 


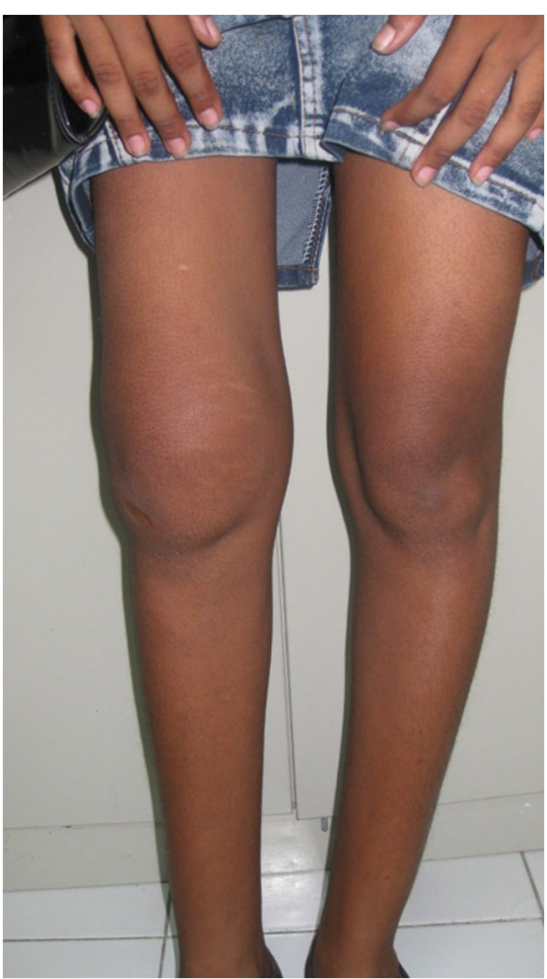

Fig. 1 Clinical photograph of both knees showing marked swelling of the right knee
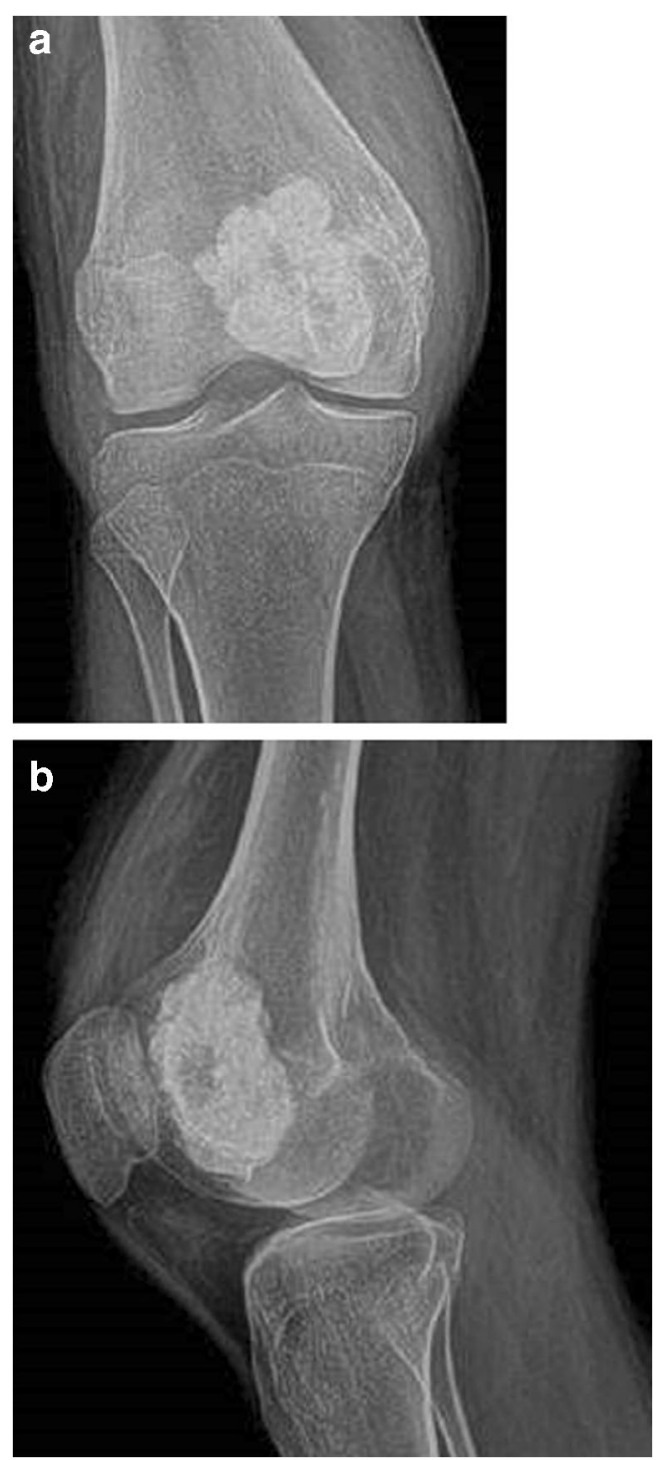

Fig. 2 a AP and $\mathbf{b}$ Lateral radiographs of the right knee

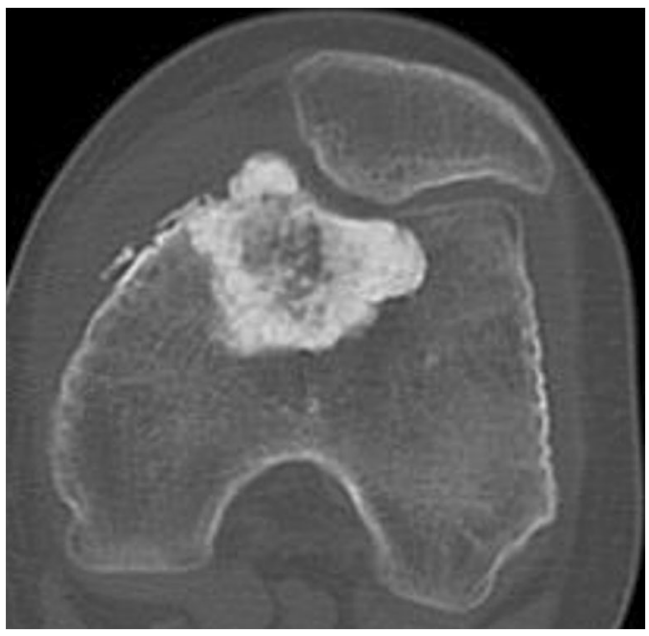

Fig. 3 Axial CT 
Fig. 4 Hematoxylin and eosin staining of the curetted specimen from surgical biopsy
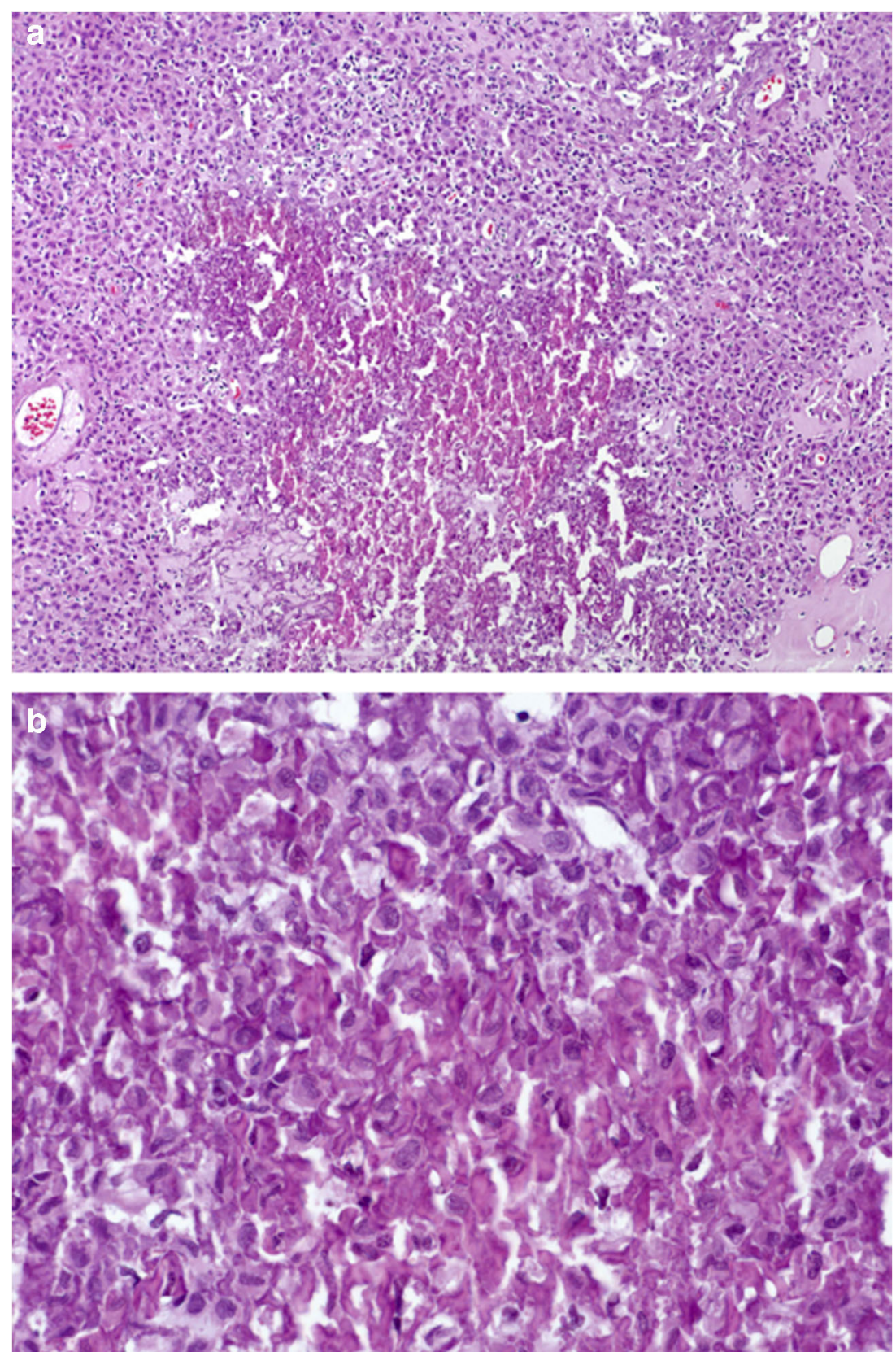


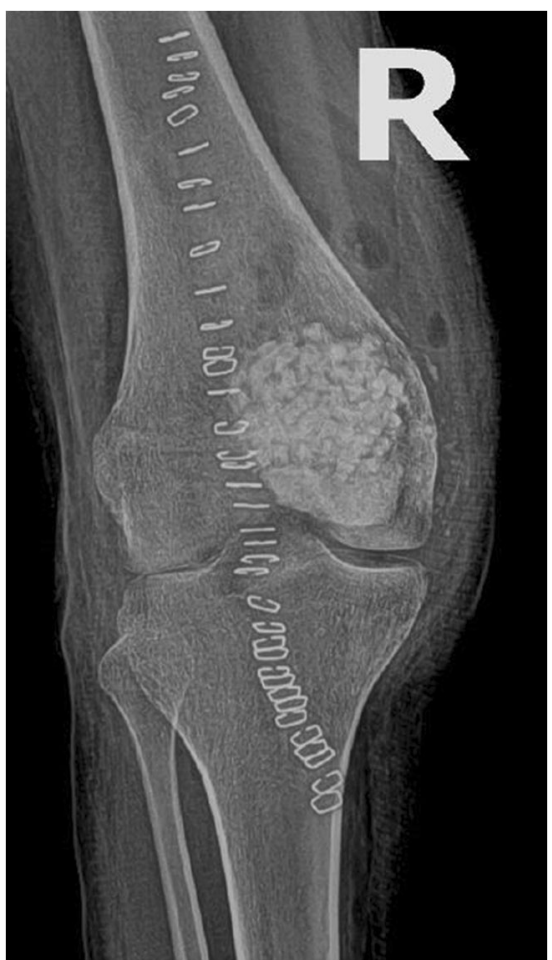

Fig. 5 AP radiograph of the right knee immediately post curetting and packing with bone graft substitute

\section{Compliance with ethical standards}

Conflict of interest The authors declare that they have no conflict of interest.

Publisher's note Springer Nature remains neutral with regard to jurisdictional claims in published maps and institutional affiliations. 